\title{
Proposal of New Medication Sensor to Prevent Forgetting to Take Medicine
}

\author{
Takuya Matsumoto ${ }^{\mathrm{a}}$, Shota Nakashima ${ }^{\mathrm{b}}$, Yuhki Kitazono, ${ }^{\mathrm{a}, *}$ \\ ${ }^{a}$ Kitakyushu National College of Technology, 5-20-1 Shii, Kokuraminami-ku, Kitakyushu-city, \\ Fukuoka 802-0985, Japan \\ ${ }^{\mathrm{b}}$ Yamaguchi University, 2-16-1 Tokiwadai, Ube-city, Yamaguchi 755-8611, Japan \\ *Corresponding Author: kitazono@kct.ac.jp
}

\begin{abstract}
The number of diabetic patients is increasing worldwide. Although treatments, such as medical diet, therapeutic exercise, and pharmacotherapy, are available to patients, they fail to show effectiveness with some patients. Some of the patients lack in daily exercises. In addition, 60 percent of the patients forget to take medicines on time. In order to solve such problems, a database system was developed to support the nursing of diabetic patients through using mobile phones. However, this system fails to function as expected due to the reason that patients are required to keep in mind of the amount of exercise they are doing daily. It is difficult for patients to keep in mind correctly of the exercise amount. Therefore, we had developed a supporting system for diabetes patients. This system can record the amount of exercise of the user automatically, and offer advice accordingly. This system can also recognize the situation of medication, and suggest the correct medication to the user. However, the system is inconvenient because the size of the medication sensor is large. In this study, we proposed a new medication sensor to prevent forgetting to take medicine on time. This medication sensor is much smaller than the previous medication sensor. This sensor can also recognize the situation of medication, and suggest the correct medication to the user.
\end{abstract}

Keywords: medication sensor, forgetting to take medicine, LED, phototransistor, magnetic sensor.

\section{Introduction}

Recently, there has been changes in lifestyle, such as lack of exercise and increasing intake of high-calorie diet ${ }^{(1)}$. If energy ingestion is higher than consumption, remind energy is stored in the body as a subcutaneous or visceral $\mathrm{fat}^{(2)}$. Therefore, Diabetes patients and obesity patients have been increasing worldwide ${ }^{(3)}$. According to "2007 National Health and Nutrition Survey" of Ministry of Health, Labour and Welfare in Japan, the number of diabetes patients in Japan is estimated to be approximately 8.9 million people ${ }^{(4)}$. Including pre-diabetes patients, it is estimated to be approximately 22.1 million people. Approximately 20 percent of Japanese is diabetes. The number of diabetes patients is expected to increase in the future ${ }^{(5)}$.

Treatments, such as medical diet, therapeutic exercise, and pharmacotherapy, are available to patients ${ }^{(6-10)}$. Medical diet is a therapy to a proper diet in order to improve the metabolic abnormalities of diabetes ${ }^{(6,7)}$. In therapeutic exercise, blood glucose levels decreases by moderate exercise $^{(6,8,9)}$. Various symptoms of diabetes can be improved in therapeutic exercise. In pharmacotherapy, drug lowers blood glucose levels ${ }^{(6,9,10)}$. Methods of pharmacotherapy are the injection of insulin and the oral hypoglycemic agents. Many patients use the oral hypoglycemic agents. It is also important for physicians to understand the status of implementation of these therapies. In therapeutic exercise, it is important to continue daily moderate exercise. However, according to "2007 National Health and Nutrition Survey" of Ministry of Health, Labour and Welfare in Japan, the percentage of people who exercise always are reached only 63.5 percent. Some of the patients lack in daily exercises ${ }^{(11)}$. On the other hand, it is important for patients to take medicines on time. However, over 60 percent of the patients forget to take medicines on time ${ }^{(12)}$. If patients do not take medicines on time, the effect 
of medicines is insufficient. In addition, it may make the state of illness worse.

In order to solve such problems, a database system was developed to support the nursing of diabetic patients through using mobile phones ${ }^{(13)}$. This system is intended to support the communication of nurse and patient. The patient can report the amount of diet, exercise, and usage of drugs. However, this system fails to function as expected due to the reason that patients are required to keep in mind of the amount of their daily exercise. It is difficult for patients to keep in mind correctly of the exercise amount. In addition, to avoid forgetting to take medicines, nurses need to always check the status of the patient medication, but this is difficult.

Therefore, we had developed some supporting systems to take medicine on time ${ }^{(14-16)}$. These systems can record the amount of exercise of the user automatically, and offer advice accordingly. These systems can also recognize the situation of medication, and suggest the correct medication to the user. However, these systems are inconvenient because the size of the medication sensor is large.

In this study, we proposed a new medication sensor to prevent forgetting to take medicine on time. The medication sensor is much smaller than the previous medication sensor. This sensor can also recognize the situation of medication, and suggest the correct medication to the user.

\section{Medication Sensor}

The structure of the medication sensor is shown in Fig. 1 and Fig. 2 shows the prototype of the sensor. The sensor judges whether the user takes medicine on time. If the user does not take medicine at the correct time, the sensor announces to the user.

\subsection{Structure}

This medication sensor is an improved product of the sensor that we have previously developed ${ }^{(16)}$. In the previous sensor, it was difficult even to carry the medicine three times a day because the size of the medication sensor is large. The new medication sensor is smaller than the previous sensor that the volume of the holder is less than one-half in comparison with the previous sensor. This new medication sensor has three small holders of the inverted triangle. There are two holes in the bottom center of each holder. From the outside of each holder, a LED and a phototransistor are set to judge whether the medicine is in

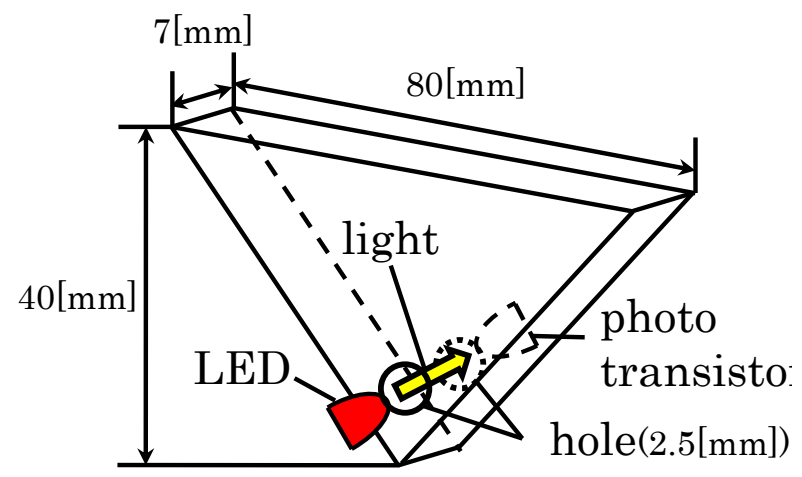

Fig. 1. The structure of the medication sensor.

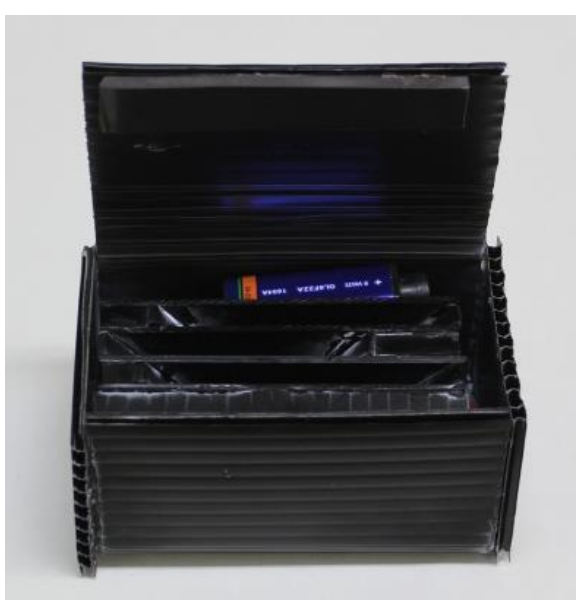

Fig. 2. The prototype of the medication sensor.

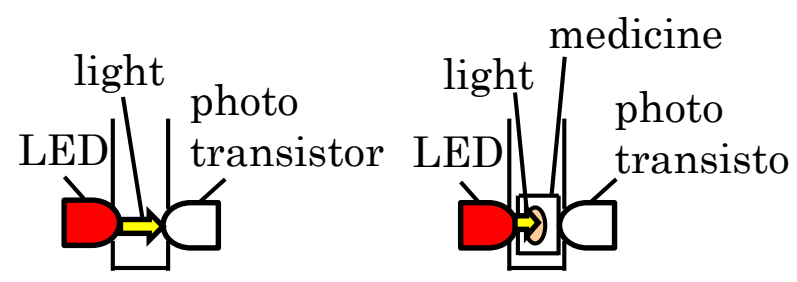

(a)There is no medicine

(b)There is the medicine

Fig. 3. The structure of recognition of the medicine.

each holder. To avoid the influence of the surround lighting, the light from the LED is modulated. Each phototransistor receives the light from each LED. From the output of the phototransistor, only the modulated frequency element is extracted by using the band pass filter. In this study, the duty ratio of laser's On/Off is set to 0.5 simply. The modulation frequency is set to $10[\mathrm{kHz}]$ that are very higher than the frequency of main turbulence such as power supply (50 or $60[\mathrm{~Hz}])$.

\subsection{Recognition of Medication}

A medicine falls down to the bottom automatically when it is put into the holder as shown in Fig. 1. The light 
from the LED is directly irradiated to the phototransistor if there is no medicine in the holder as shown in Fig. 3a. However, if there is a medicine in the holder, the light from the LED is reflected by medicine and the light received by phototransistor is reduced as shown in Fig. 3b. By the presence of medicine, the intensity of the light received by the phototransistor is different. If the output is higher than the threshold voltage $V_{M}$, this sensor recognizes there is a medicine in the holder. Otherwise, the sensor recognizes there is no medicine in the holder. The threshold voltage $V_{M}$ is determined in 3.1 .

When the sensor is placed upside down, the unit cannot accurately determine the presence of medicine. However, while there is no opening and closing of the lid, the presence of medicine does not change and by attaching a magnet to the lid, the unit can recognizes the opening and closing of the lid by using a magnetic sensor. Furthermore, This unit only recognizes the presence of medicine immediately after the lid is closed. Therefore, this unit can recognize the presence of medicine correctly because the sensor is not upside down immediately after the lid is closed. It is sufficient to light the LED only after the lid is closed. This function also contributes to energy savings of this unit.

\subsection{Announcement about Medication}

Prior to use the medication sensor, the start and end time of taking medicine is input by the user. The medication sensor announces the following from the output and the time entered by the user. Therefore, the medication sensor can encourage the proper medication to the diabetes patients.

i. In the start time of taking medicine, the medication sensor announces that there is the time of taking medicine now.

ii. If the user tries to take medicine before the start time of taking medicine, the medication sensor announces to stop taking medicine.

iii. If the user does not take medicine between the time entered by the user, the medication sensor announces that the user has forgotten to take medicine.

\section{Experiment}

\subsection{Decision of Threshold Voltage $V_{M}$}

Firstly, the threshold voltage $V_{M}$ for the recognition of

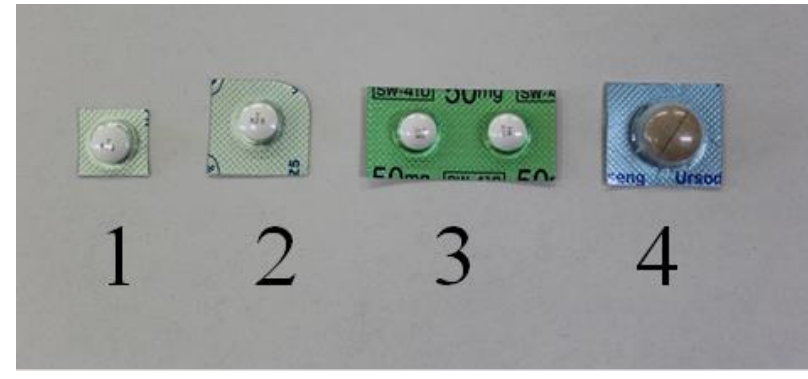

Fig. 4. Medicines used in the experiment.

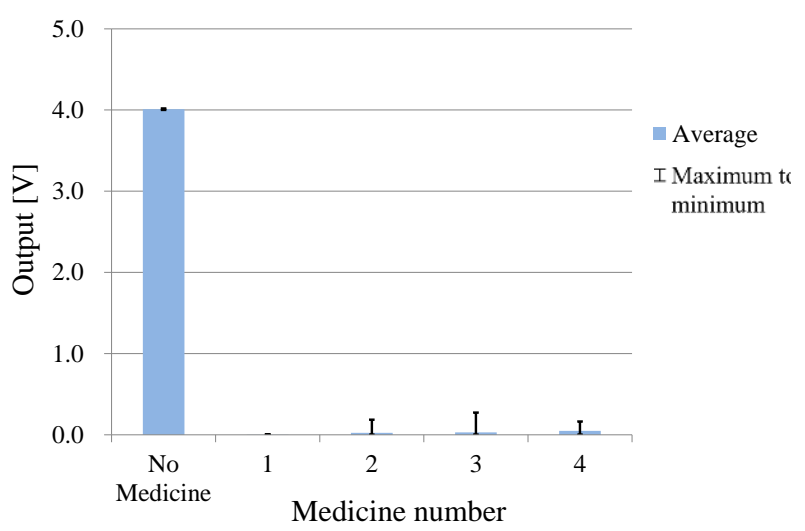

Fig. 5. The output of the medication sensor.

medication was decided in this experiment. The medicine was put into the medication sensor and the output of the medication sensor was measured to the sensor. The output of the medication sensor was also measured when there was no medicine in the sensor.

Four types of medicines with different sizes and shapes are used as shown in Fig. 4. It was tried 10 times for each condition. Figure 5 shows the average output and the range from minimum to maximum. According to the results, the recognition rate of 100 percent was obtained at the range of $0.3 \sim 3.9[\mathrm{~V}]$ of $V_{M}$. Therefore, $V_{M}$ was decided 2.0 [V].

\subsection{Operation Verification}

Secondly, we did operation verification of recognition of medicine using threshold voltage $\boldsymbol{V}_{\boldsymbol{M}}$ decided in 3.1. One medicine was fallen into each holder at random 10 times for each medicine as shown in Fig. 4. As the result, the medication sensor recognized all medicine put into the holder and the recognition rate is 100 percent.

\subsection{Announcement about Medication}

Thirdly, the announcement from the medication sensor was verified. In advance, the user put a medicine in the 
medication sensor and inputted the start and end time of taking medicine. The experiment was performed in the following 3 conditions.

i. The user took medicine between the start and end time of taking medicine.

ii. The user tried to take medicine before the start time of taking medicine.

iii. The user does not take medicine between the time entered by the user.

As a result, the medication sensor behaved as follows.

i. In the start time of taking medicine, the medication sensor announced that there is the time of taking medicine now.

ii. The medication sensor announced to stop taking medicine.

iii. The medication sensor announced that the user had forgotten to take medicine.

Therefore, it is confirmed that the medication sensor announced to the user according to the situation of the medication.

\section{Conclusions}

In this study, we proposed a new medication sensor for patients who need to take medicine on time. This medication sensor is much smaller than the previous medication sensor. By using phototransistor, this medication sensor was able to recognize the situation of medication, and suggest the correct medication to the user. Therefore, this sensor is useful in the treatment.

As future prospects, we are planning to develop a system to consider the dose method.

\section{References}

(1) Takehiro Nozaki : "Diabetes, Obesity and Depression", Japanese Journal of Psychosomatic Medicine, Vol. 51, No. 10, pp. 886-895, 2011

(2) Toru Fushiki : "Strategic Reinforcement of Body Energy Expenditure by Foodstuffs :with Non-Pungent Hot Pepper", The Japanese Journal of Taste and Smell Research, Vol. 13, No. 2, pp. 169-174, 2006

(3) Committee for Healthy Japan 21 : "Final Assessment of Healthy Japan 21”, p. 16-19, 2011

(4) Ministry of Health, Labour and Welfare : "2007 National Health and Nutrition Survey”, p. 45, 2010

(5) Mitsuharu, Kumai, Yoshika Suzaki, and Hiromi Ariyoshi : “A Psychological Pathogenesis of Patient with Type 2 Diabetes :A Comparison with Major Depression Using a Multi-faceted Life Stress Questionnaire”, Vol. 13, No. 1, pp. 5-11, 2010

(6) Yorimitsu Furukawa : "Exercise Outline of Diabetes Mellitus and Obesity", The Journal of Japan Academy of Health Sciences, Vol. 51, No. 10, pp. 886-895, 2011

(7) Chihiro Inaba, Sumie Suzuki, and Itoko Tobita : "Analysis of Intervention Concerning Self Management of a Diet Therapy Regimen for Patients with Diabetes Mellitus", The 26th Annual Convention of the Japanese Association for Behavior Analysis, No. 26, p. 50, 2008

(8) Tsutomu Kashi, Takao Nakamura, and Masataka Kusunoki : "Effect of Peripheral Muscle Contraction on Insulin Sensitivity", IEICE Technical Report MBE, Vol. 102, No. 137, pp. 33-36, 2002

(9) Takeo Itoh, Junko Kajikuri, Yoshie Ito, and Yasushi Shibayama : "Rehabilitation and Pharmacological Therapy for Diabetes”, Physical Therapy Japan, Vol. 37, No. 4, pp. 247-250, 2010

(10) Katsuyoshi Tojo : "Recent Progress in the Medical Therapy of Acromegaly", Japanese Journal of Neurosurgery, Vol. 16, No. 9, pp. 686-698, 2007

(11) Ministry of Health, Labour and Welfare : "2010 National Health and Nutrition Survey”, p. 128, 2012

(12) Nobuaki Kutsuma, Katunori Yamaura, Megumi Nakayama, Mihoko Namiki, Keiko Akiba, Soichiro Kimura, Hideo Ueda, Sachihiko Numajiri, Daisuke Kobayashi, and Yasunori Morimoto : "Practice for Promoting Proper Use of Reusing the Leftover Medicine in the Outpatients of the Chronic Disease", Journal of the Pharmaceutical Society of Japan, Vol. 124, No. 6, pp. 355-364, 2004

(13) Kazuhiro Ikemoto, Izumi Kawamura, Dasisuke Morihama, Takuya Yoshihiro, Mumiko Mori, Sachiko Kagawa, Yasuhisa Yamamoto, and Masaru Nakagawa : "A Database System to Support Nursing Diabetic People Using Mobile Phones", IPSJ SIG Technical Reports DBS GN, Vol. 2006, No. 9, pp. 197-202, 2006

(14) Yuhki Kitazono, Takuya Matsumoto, Shota Nakashima, Shiyuan Yang, and Seiichi Serikawa : "A Monitoring System to Prevent Forgetting to Take Medicines on Time Using Funnel-Shaped Sensors", Applied Mechanics and Materials, Vol. 103, pp. 606-610, 2011

(15) Yuhki Kitazono, Xianglan Zheng, Shota Nakashima, Shiyuan Yang, and Seiichi Serikawa : "Proposal of system for prevention of forgetting to take medicines using capacity", Procedia - Social and Behavioral 
Sciences, Vol. 2, Iss. 1, pp. 193-197, 2010

(16) Yuhki Kitazono, Takuya Matsumoto, Takakazu Mouri, and Shota Nakashima : "Development of System to Support Diabetes Treatment", Journal of the Institute of Industrial Applications Engineers, Vol. 1, No. 1, pp. 29-34, 2013 